\title{
Les phosphoinositides, des lipides acteurs essentiels du trafic intracellulaire
}

\author{
Dimitri L. Bertazzi, Johan-Owen De Craene, Séverine Bär, Myriam Sanjuan-Vazquez, Matthieu A. Raess \\ et Sylvie Friant \\ Département de Génétique Moléculaire et Cellulaire, UMR 7156, Université de Strasbourg et CNRS, \\ 67084 Strasbourg, France \\ Auteur correspondant : Sylvie Friant, s.friant@unistra.fr
}

Reçu le 19 novembre 2014

Résumé - Les phosphoinositides sont des lipides impliqués dans le transport vésiculaire des protéines entre les différents compartiments. Ils agissent par le recrutement et/ou l'activation de protéines effectrices et sont de ce fait impliqués dans la régulation de différentes fonctions cellulaires telles que le bourgeonnement vésiculaire, la fusion ou la dynamique des membranes et du cytosquelette. Bien que présents en faible concentration dans les membranes, leur rôle est indispensable à la survie des cellules et doit être régulé avec précision. Le contrôle de leur fonction se fait par la phosphorylation/déphosphorylation des positions D3, D4 et/ou D5 de leur anneau inositol par des kinases et phosphatases spécifiques des différentes membranes intracellulaires. Ces enzymes sont en partie conservées entre la levure et l'Homme et leur perte de fonction peut entraîner des maladies génétiques graves comme les myopathies.

Mots clés : Lipides / trafic intracellulaire / phosphoinositide / phosphatase / kinase

Abstract - Phosphoinositides: lipidic essential actors in the intracellular traffic.

Phosphoinositides (PPIn) are lipids involved in the vesicular transport of proteins between the different intracellular compartments. They act by recruiting and/or activating effector proteins and are thus involved in crucial cellular functions including vesicle budding, fusion and dynamics of membranes and regulation of the cytoskeleton. Although they are present in low concentrations in membranes, their activity is essential for cell survival and needs to be tightly controlled. Therefore, phosphatases and kinases specific of the various cellular membranes can phosphorylate/dephosphorylate their inositol ring on the positions D3, D4 and/or D5. The differential phosphorylation determines the intracellular localisation and the activity of the PPIn. Indeed, non-phosphorylated phosphatidylinositol (PtdIns) is the basic component of the PPIn and can be found in all eukaryotic cells at the cytoplasmic face of the ER, the Golgi, mitochondria and microsomes. It can get phosphorylated on position D4 to obtain PtdIns4P, a PPIn enriched in the Golgi compartment and involved in the maintenance of this organelle as well as anterograde and retrograde transport to and from the Golgi. PtdIns phosphorylation on position D3 results in PtdIns3P that is required for endosomal transport and multivesicular body (MVB) formation and sorting. These monophosphorylated PtdIns can be further phosphorylated to produce bisphophorylated PtdIns. Thus, PtdIns(4,5) $P_{2}$, mainly produced by PtdIns4P phosphorylation, is enriched in the plasma membrane and involved in the regulation of actin cytoskeleton and endocytosis. PtdIns(3,5) $P_{2}$, mainly produced by PtdIns3P phosphorylation, is enriched in late endosomes, MVBs and the lysosome/vacuole and plays a role in endosome to vacuole transport. $\operatorname{PtdIns}(3,4) P_{2}$ is absent in yeast, cells and mainly produced by PtdIns $4 P$ phosphorylation in human cells; PtdIns $(3,4) P_{2}$ is localised in the plasma membrane and plays an important role as a second messenger by recruiting specific 
protein kinases (Akt and PDK1). Finally the triple phosphorylated PPIn, PtdIns $(3,4,5) P_{3}$ also absent in yeast, is produced by the phosphorylation of PtdIns $(3,4) P_{2}$ and localized at the plasma membrane of human cells where it binds proteins via their PH domain. Interaction partners include members of the Arf (ADPribosylation factors) family, PDK1 (Phosphoinositide Dependent Kinase 1) and Akt. Therefore this last PPIn is essential for the control of cell proliferation and its deregulation leads to the development of numerous cancers. In conclusion, the regulation of PPIn phosphorylation/dephosphorylation is complex and needs to be very precisely regulated. Indeed phosphatases and kinases allow the maintenance of the equilibrium between the different forms. PPIn play a crucial role in numerous cellular functions and a loss in their synthesis or regulation results in severe genetic diseases.

Key words: Lipids / intracellular trafficking / phosphoinositides / phosphatase / kinase

$\begin{array}{ll}\text { Abréviations } & \\ \text { ENTH } & \text { Epsin N-Terminal Homology } \\ \text { ESCRT } & \text { Endosomal Sorting Complex } \\ & \text { Required for Transport } \\ \text { FYVE } & \text { Fab1, YGL023, Vps27, EEA1 } \\ \text { MVB } & \text { MultiVesicular Body } \\ \text { Osh1 } & \text { Oxysterol binding protein } \\ & \text { homologue 1 } \\ \text { PC } & \text { PhosphatidylCholine, } \\ \text { PE } & \text { PhosphatidylEthanolamine } \\ \text { PH } & \text { Pleckstrin Homology } \\ \text { PHD } & \text { Plant HomeoDomain finger } \\ \text { PIKfyve } & \text { PtdIns3P 5-Kinase (humaine) } \\ \text { Pis1 } & \text { PtdIns synthase 1 } \\ \text { PPIn } & \text { PolyPhosphoInositides } \\ \text { PS } & \text { PhosphatidylSérine, } \\ \text { PtdIns } & \text { PhosphatidylInositol } \\ \text { PtdIns3P } & \text { PhosphatidylInositol 3-phosphate } \\ \text { PtdIns }(3,4) \mathrm{P}_{2} & \text { PhosphatidylInositol 3, } \\ & \text { 4-bisphosphate } \\ \text { PtdIns }(3,4,5) \mathrm{P}_{3} & \text { PhosphatidylInositol 3, } \\ & \text { 4,5-trisphosphate } \\ \text { PTEN } & \text { Phosphatase and TENsin } \\ & \text { homolog } \\ \text { SM } & \text { SphingoMyéline } \\ \text { Vps34 } & \text { Vacuolar Protein Sorting 34 } \\ & \end{array}$

\section{Le métabolisme des phosphoinositides membranaires}

\subsection{Les lipides, constituants majoritaires des membranes}

Les membranes ne sont pas des structures figées mais dynamiques dont les propriétés physico-chimiques font partie des facteurs ayant permis l'apparition de la vie. En effet, l'environnement, au sens large, est caractérisé par des variations de température, d'humidité, de $\mathrm{pH}$, d'ensoleillement, de pression osmotique ou de source d'énergie, autant de facteurs auxquels tout organisme vivant doit s'adapter afin de conserver un équilibre interne. C'est pour gagner en stabilité face à toutes ces fluctuations incontrôlables que la cellule s'est organisée de façon à limiter les variations intracellulaires. La membrane plasmique est le premier rempart permettant de séparer le milieu extérieur du cytoplasme. Sa composition lui assure une protection mécanique et un contrôle des échanges avec l'extérieur grâce à une perméabilité très sélective.

La membrane plasmique s'organise en deux feuillets de phospholipides, la bicouche, dans laquelle s'enchâssent les macromolécules protéiques, stérols et glycolipides. Les phospholipides qui constituent cette bicouche sont amphiphiles avec un groupe hydrophile (tête) lié à un groupe hydrophobe (queue). Au sein de la bicouche, les groupes hydrophobes se font face, générant un espace hydrophobe entre les deux feuillets membranaires. Cette propriété est très importante pour l'ancrage dans la membrane de molécules lipidiques (stérols ou céramides), de protéines transmembranaires ou de protéines ayant une ancre lipidique. La composition en lipides des membranes varie selon les organismes (eucaryotes ou procaryotes), les types cellulaires (au sein des tissus d'un même organisme pluricellulaire), le type de membrane (plasmique, réticulum endoplasmique, endosomes, appareil de Golgi, mitochondries et autres compartiments intracellulaires) et même l'état de la cellule (au repos ou en réponse à un stress/stimulus) (Spector \& Yorek, 1985; Zinser et al., 1991).

Les membranes sont composées principalement de cinq phospholipides : la phosphatidylcholine (PC), la phosphatidyléthanolamine (PE), la phosphatidylsérine (PS), le phosphatidylinositol (PtdIns) et la sphingomyéline (SM). Les stérols, bien que présents dans les membranes cellulaires, ne forment pas la membrane par eux-mêmes mais modulent sa 
fluidité, essentielle pour la diffusion latérale des molécules dans la bicouche. Le double feuillet lipidique des membranes présente également une distribution asymétrique des phospholipides entre les feuillets interne et externe, qui résulte en partie de la diffusion verticale entre les deux feuillets par flip-flop, un mécanisme peu efficace.

Si la membrane plasmique joue un rôle essentiel de barrière sélective, on retrouve dans les cellules eucaryotes d'autres structures membranaires intracellulaires telles que les organites et les vésicules de transport. L'organisation de ces membranes ainsi que leur composition diffèrent selon la nature du compartiment. En effet, le réticulum endoplasmique, l'appareil de Golgi, les lysosomes (la vacuole chez les levures), les endosomes et les vésicules de transport sont entourés d'une seule bicouche lipidique. L'espace à l'intérieur de ces compartiments intracellulaires est nommé lumen ou lumière. En revanche, le noyau, les mitochondries, les chloroplastes des végétaux chlorophylliens et les vésicules autophagiques sont délimités par une double bicouche lipidique. Chacun de ces compartiments intracellulaires s'acquitte de fonctions bien précises nécessaires à la vie de la cellule (Spector \& Yorek, 1985). Cette dernière doit donc chorégraphier le transport vésiculaire de protéines de manière spatiotemporelle entre ces différents compartiments. Il est par conséquent indispensable pour la cellule de discriminer un compartiment intracellulaire d'un autre. Ceci passe par l'attribution d'une identité propre à chacun des organites allant même jusqu'à distinguer les faces «cis» des faces «trans» de l'appareil de Golgi. Ces « cartes d'identité » propres à chaque compartiment intracellulaire sont essentiellement définies par les molécules tapissant le feuillet cytoplasmique des membranes. Parmi ces molécules, les lipides membranaires, et plus particulièrement les phosphoinositides jouent un rôle majeur dans la régulation spatiotemporelle de différents processus cellulaires tels que la dynamique du cytosquelette, le trafic membranaire, la prolifération et la survie cellulaire.

\subsection{Les phosphoinositides, des médiateurs lipides}

Les polyphosphoinositides (PPIn) sont des constituants mineurs des membranes cellulaires, représentant moins de $10 \%$ des phospholipides cellulaires (Payrastre et al., 2001). Les PPIn sont composés d'une molécule de glycérol estérifiée en position SN1 et SN2 par deux chaînes d'acides gras et reliée en position SN3 à un anneau inositol par l'intermédiaire d'un groupement phosphate (Payrastre et al., 2001). Chez l'Homme, les acides gras les plus courants dans le PtdIns sont l'acide stéarique (18:0) en position SN1 et l'acide arachidonique (20:4) en SN2 (Marcus et al., 1969). Chez la levure Saccharomyces cerevisiae, la nature des acides gras est légèrement différente; on retrouve ainsi une majorité d'acide oléique (18:1), d'acide palmitoléique (16:1) et d'acide palmitique (16:0) (Trevelyan, 1966). Etant donné que les diverses espèces de PPIn dérivent du PtdIns, on estime que leurs compositions en acides gras sont les mêmes que celles du PtdIns.

L'anneau inositol des PPIn est un polyol de cyclohexane dont les position D3, D4 et D5 sont phosphorylables, générant potentiellement sept PPIn différents (figure 1) : le phosphatidylinositol 3-phosphate (PtdIns3P), le PtdIns4P, le PtdIns5 P, le PtdIns 3,4-bisphosphate $\left(\operatorname{PtdIns}(3,4) P_{2}\right)$, le $\operatorname{PtdIns}(3,5) P_{2}$, le PtdIns $(4,5) P_{2}$ et le PtdIns 3,4,5-trisphosphate $\left(\operatorname{PtdIns}(3,4,5) P_{3}\right)$. Malgré leur faible concentration dans les membranes, les PPIn jouent un rôle essentiel dans le recrutement et/ou l'activation de protéines effectrices. De plus, leur présence dans une membrane donnée et leurs niveaux varient grâce aux lipides kinases et phosphatases spécifiques des différentes membranes, permettant ainsi la régulation spatiotemporelle de divers événements tels que le bourgeonnement, la fusion ou la dynamique des membranes (Payrastre et al., 2001).

\subsection{Le phosphatidylinositol Ptdlns, précurseur des phosphoinositides}

Le PtdIns, point de départ du métabolisme des PPIn, est un phospholipide ubiquitaire dans les cellules eucaryotes mais dont la proportion varie selon le type de membrane. En effet, les PPIn sont tous métabolisés directement ou séquentiellement à partir du PtdIns (figure 1). Chez la levure S. cerevisiae, le PtdIns est synthétisé par la PtdIns synthase 1 (Pis1) au niveau de la face cytoplasmique de la membrane du réticulum endoplasmique, de l'appareil de Golgi, des mitochondries et des microsomes (Nikawa \& Yamashita, 1984). Chez l'Homme, cette synthèse est catalysée par l'homologue de Pis1, la PtdIns Synthase qui présente une distribution similaire à celle de Pis1 chez la levure (Antonsson, 1994).

Le PtdIns est également recyclé par la conversion du PtdIns3P, du PtdIns4 $P$ et du PtdIns5 $P$ en PtdIns par l'action de lipides phosphatases (figure 1). Chez l'Homme, les myotubularines (MTM1, MTMR14,6,7) sont des 3-phosphatases déphosphorylant spécifiquement la position D3 et réalisant, entre autres, la conversion du PtdIns3P en PtdIns (Laporte et al., 2003). Les autres phosphatases sont moins spécifiques car elles sont capables de déphosphoryler le PtdIns3P en PtdIns, comme la PPIn phosphatase SAC1, qui déphosphoryle également le PtdIns $4 P$ et probablement le PtdIns $5 P$ en PtdIns (Liu \& Bankaitis, 2010). La levure S. cerevisiae présente également une phosphatase spécifique de la 


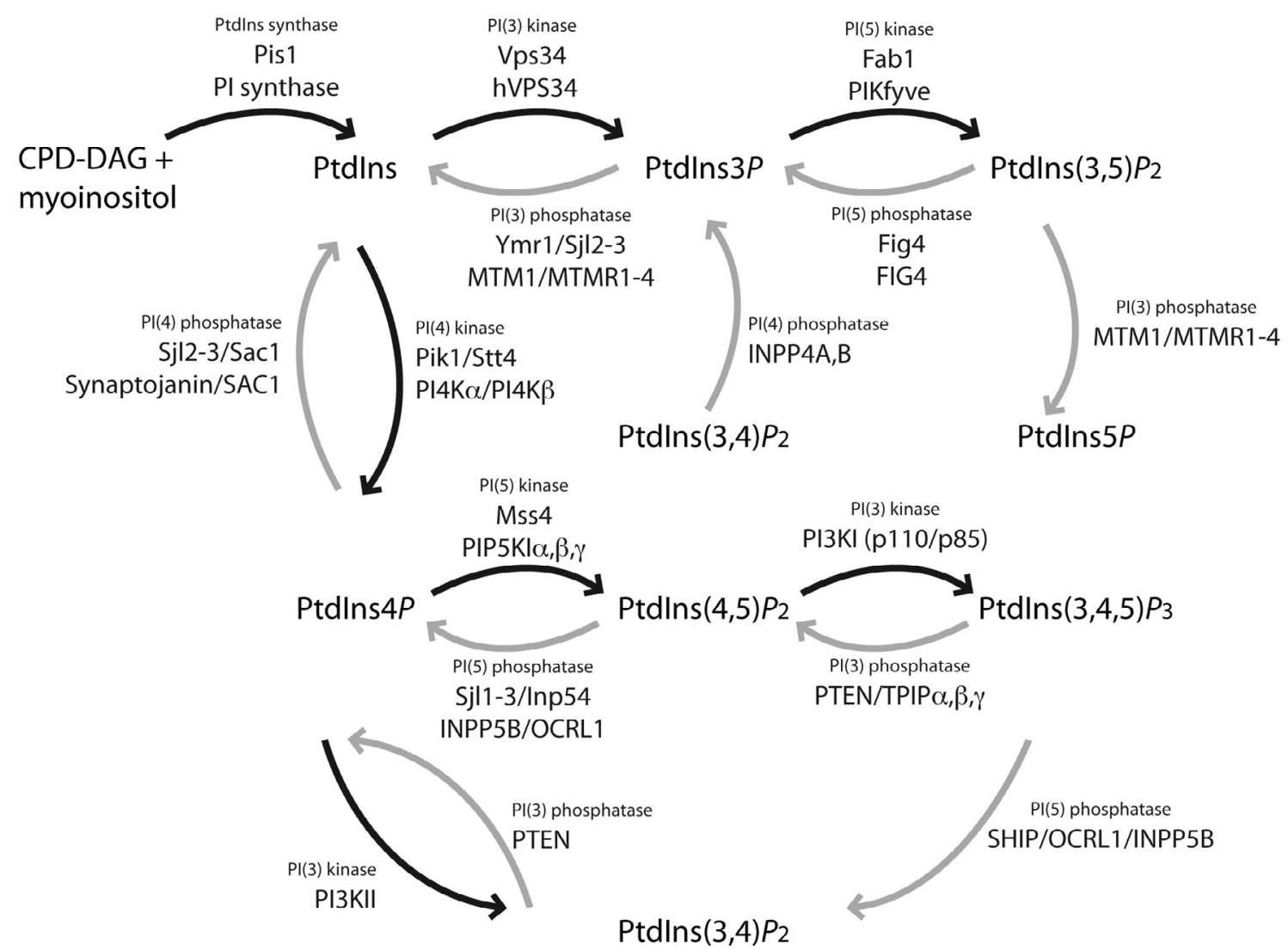

Fig. 1. Synthèse des phosphoinositides chez la levure S.cerevisiae et chez l'Homme avec les enzymes impliquées. Les réactions de phosphorylation sont représentées par des flèches noires et celles de déphosphorylation par des flèches grises. Le nom des enzymes de levure (quand elles existent) est indiqué en haut et leur homologue chez l'Homme est noté en-dessous.

position D3 avec son unique homologue de myotubularine Ymr1. Cette activité sur le PtdIns3P est partagée avec des phosphatases plus générales que sont les Synaptojanin-like protein 1 et 2 (Sjl2 et Sjl3) et Sac1, aussi capables de convertir le PtdIns $4 P$ en PtdIns (Parrish et al., 2004).

\section{Le Ptdlns4P, un effecteur clé du trafic au niveau de l'appareil de Golgi}

\subsection{Synthèse du Ptdlns4P}

Le PtdIns4P représente environ $30 \%$ des PPIn totaux chez la levure, et approximativement $45 \%$ chez l'Homme (Payrastre et al., 2001). Il est enrichi au niveau de l'appareil de Golgi où il est majoritairement généré par phosphorylation du PtdIns par des PI 4-kinases (De Matteis et al., 2002). Il peut aussi résulter de la déphosphorylation du $\operatorname{PtdIns}(4,5) P_{2}$ et du PtdIns $(3,4) P_{2}$ par des PI 5-phosphatases et des PI 3-phosphatases, respectivement (figure 1). Chez S. cerevisiae, les PI 4-kinases Pik1 et Stt4 réalisent la conversion du PtdIns en PtdIns4P au niveau de l'appareil de Golgi et de la membrane plasmique, respectivement (Audhya et al., 2000). La déphosphorylation du PtdIns $(4,5) P_{2}$ en PtdIns4P est orchestrée par les 5-phosphatases Inp51/Sj11, Inp52/Sj12, Inp53/Sj13 et Inp54 (figure 1) (Liu \& Bankaitis, 2010). Chez l'Homme, les PPIn 4-kinases $\mathrm{PI} 4 \mathrm{~K} \alpha$ et $\mathrm{PI} 4 \mathrm{~K} \beta$ synthétisent le $\operatorname{PtdIns} 4 P$ à partir du précurseur PtdIns. Par ailleurs, le PtdIns4Ppeut être synthétisé à partir du $\operatorname{PtdIns}(3,4) P_{2}$ par la 3-phosphatase $\mathrm{PTEN}$ ou à partir du $\operatorname{PtdIns}(4,5) P_{2}$ par les 5-phosphatases OCRL1 (Occulo-cerebro-renal syndrome protein 1), INPP5B (Inositol Polyphosphate Phosphatase 5B) et les synaptojanines 1 et 2 (figure 1) (Liu \& Bankaitis, 2010). Des mutations dans OCRL1 sont à l'origine du syndrome occulo-cérébro-rénal de Lowe.

\subsection{Rôle physiologique du Ptdlns4P}

Le PtdIns $4 P$ a pendant longtemps été considéré comme un simple précurseur d'autres PPIn. Chez 
la levure, le PtdIns4P est présent dans deux compartiments distincts : la membrane plasmique et le réseau trans-golgien (Audhya et al., 2000). L'appareil de Golgi constitue un carrefour central dans le trafic membranaire, où les protéines et les membranes de divers compartiments intracellulaires s'échangent, nécessitant donc la régulation spatio-temporelle très précise de ces processus. Le PtdIns $4 P$ serait impliqué dans le maintien de l'identité de l'appareil de Golgi, afin de préserver son rôle dans le trafic intracellulaire. Ainsi, chez la levure, le PtdIns4P a été montré comme ayant une fonction dans le transport de protéines : le transport antérograde à partir du réseau transgolgien et le transport rétrograde vers l'appareil de Golgi (Audhya et al., 2000). De plus, chez la levure le PtdIns4 $P$ est important pour la formation des vésicules de sécrétion allant du Golgi vers la membrane plasmique (Mizuno-Yamasaki et al., 2010). De nombreuses protéines interagissant avec le PtdIns4P ont été identifiées et sont localisées à l'appareil de Golgi (Lemmon, 2003). Par exemple, la protéine de levure Osh1 (Oxysterol binding protein homologue), requise pour le transport des stérols, est localisée à l'appareil de Golgi via son domaine PH (Pleckstrin Homology) qui lie le PtdIns4P (Levine \& Munro, 2002).

L'homologue humain de Osh1, la protéine OSBP (OxySterol Binding Protein), également impliquée dans le transport intracellulaire des stérols, se localise au niveau du Golgi grâce à l'interaction entre son domaine $\mathrm{PH}$ et le PtdIns4P (Levine \& Munro, 2002). Cependant, si cette interaction est essentielle à la localisation de OSBP, elle n'est pas suffisante puisqu'elle nécessite également l'interaction avec une GTPase golgienne (ADP-Ribosylation Factor ou ARF) (Levine \& Munro, 2002). Deux autres protéines, FAPP1 et FAPP2 (Four-Phosphate-Adaptor Protéine 1 et 2) qui régulent le trafic membranaire golgien, sont également en complexe avec ARF et interagissent avec le PtdIns4P via leur domaine PH (Lemmon, 2003). Cette interaction protéine-lipide jouerait un rôle dans le transport vésiculaire antérograde du Golgi vers la membrane plasmique en contrôlant la production de vésicules bourgeonnant depuis l'appareil de Golgi (Godi et al., 2004).

\section{Le Ptdlns3P, un lipide essentiel au trafic membranaire au niveau des endosomes}

\subsection{Synthèse du Ptdlns3P}

Le PtdIns3P représente environ $30 \%$ des PPIn totaux de levure et est présent en quantité équivalente aux PtdIns4P. Par ailleurs chez l'Homme, il représente moins de $15 \%$ des PPIn mono-phosphorylés et est largement minoritaire par rapport au PtdIns4P (Payrastre et al., 2001). Le PtdIns3P est produit par phosphorylation du PtdIns en position D3 de l'inositol ou par déphosphorylation du PtdIns $(3,4) P_{2}$ ou du $\operatorname{PtdIns}(3,5) P_{2}$ (figure 1).

Chez la levure $S$. cerevisiae, une seule enzyme catalyse spécifiquement et uniquement la phosphorylation du PtdIns en PtdIns3P, la lipide kinase Vps34 ( $\mathrm{Va}$ cuolar Protein Sorting 34) (Herman \& Emr, 1990; Schu et al., 1993). L'activité de Vps34 est régulée par la protéine kinase Vps15 et est indispensable à la formation du MVB (MultiVesicular Body ou corps multivésiculaire). De façon intéressante, il a été montré que la régulation positive de Vps34 par Vps15 est stimulée par l'interaction directe entre les sept répétitions WD (Trp-Asp) situées dans la région Cterminale de cette dernière et la sous-unité $\mathrm{G} \alpha$ de la protéine Gpa1. Le domaine C-terminal de Vps15 se comporterait ainsi comme la sous-unité $\beta$ de la protéine G. Ainsi, il y aurait un couplage entre la signalisation médiée par les protéines $\mathrm{G}$ au niveau de la membrane plasmique et le tri des récepteurs de cette signalisation au niveau des endosomes. De plus, l'interaction du complexe Vps34-Vps15 avec Gpa1 stimule la production de PtdIns3P aux endosomes (Slessareva et al., 2006).

Le génome humain code pour huit lipide-kinases capables de produire du PtdIns3P et regroupées en trois classes, selon leurs spécificités de substrat et leur homologie (Vanhaesebroeck et al., 2001) :

- Deux phosphoinositides 3-kinases de classe I (PI3K I) qui phosphorylent surtout le $\operatorname{PtdIns}(4,5) P_{2}$ en $\operatorname{PtdIns}(3,4,5) P_{3}$.

- Trois phosphoinositides 3-kinases de classe II (PI3K II) qui phosphorylent surtout le PtdIns4P en $\operatorname{PtdIns}(3,4) P_{2}$.

- La phosphoinositide 3-kinase de classe III, qui est l'homologue de la protéine de levure Vps34. Tout comme Vps34, la PI3K III humaine est spécifique du PtdIns et est par conséquent probablement à l'origine de l'essentiel du PtdIns3P cellulaire. La sous-unité régulatrice de $\mathrm{hVps} 34$ est la protéine p150, l'homologue de Vps15 de levure (Panaretou et al., 1997). Une étude phylogénétique a mis en évidence la coévolution de l'unique PI3K III et de sa sous-unité régulatrice Vps15 chez la plupart des eucaryotes, de la levure à l'Homme en passant par les amibes et les parasites (Lecompte et al., 2008).

Chez la levure, le PtdIns3P est également synthétisé par les lipides phosphatases Fig4 (Factor Induced Gene 4, également appelée Sac3), Sj12/Inp52, Sj13/Inp53 et Sac1. Elles possèdent toutes un domaine SAC catalytique permettant de déphosphoryler différents PPIn dont le $\operatorname{PtdIns}(3,5) P_{2}$ en PtdIns3P (Liu \& Bankaitis, 2010). Fig4 est la seule PtdIns 5-phosphatase spécifique du $\operatorname{PtdIns}(3,5) P_{2}$ 
(Gary et al., 2002). L'homologue humain FIG4/SAC3 remplit des fonctions cellulaires similaires à Fig4. Des mutations dans le gène humain FIG4 sont à l'origine du syndrome de Charcot-Marie-Tooth type 4J, une maladie neuromusculaire de forme récessive qui se traduit par une démyélinisation des axones (Liu \& Bankaitis, 2010). Avec MTMR2, FIG4 est donc le deuxième gène codant pour une lipide phosphatase impliquée dans une maladie neuromusculaire.

\subsection{Rôle physiologique du Ptdlns3P}

Dans les cellules de levure et de mammifères, le PtdIns3P est enrichi au niveau de la membrane des endosomes précoces et à la membrane des vésicules internes des endosomes tardifs (ou corps multivésiculaires, ou MVB) (Gillooly et al., 2000). Au niveau des endosomes précoces, il joue un rôle central dans le recrutement de protéines effectrices telles que la protéine de levure Vps27 ou son homologue humaine Hrs (Hepatocyte growth factor-Regulated tyrosine kinase Substrate), deux protéines du complexe ESCRT-0 (Endosomal Sorting Complex Required for Transport) impliquées dans le tri endosomal des protéines et la formation du MVB (Henne et al., 2011). Ces deux protéines comportent un domaine FYVE (Fab1, YGL023, Vps27, et EEA1) liant le PtdIns3P endosomal et un motif de recrutement du complexe ESCRT-1 (Gruenberg \& Stenmark, 2004). Ceci permet de recruter les deux autres complexes ESCRT-2 et 3 dont l'action concertée permet l'internalisation des protéines membranaires dans les vésicules internes du MVB (Henne et al., 2011). Cette internalisation est indispensable à l'arrêt des cascades de signalisation médiées par des récepteurs transmembranaires et à l'adressage de protéines membranaires à la vacuole/lysosome.

La protéine adaptatrice humaine EEA1 (Early Endosomal Antigen 1) se lie également au PtdIns3P avec une forte affinité via son domaine FYVE et régule les processus de fusion membranaire entre endosomes en recrutant la GTPase Rab5 aux endosomes (Gruenberg \& Stenmark, 2004).

De même, la PtdIns3P 5-kinase Fab1 (S. cerevisiae) ou PIKfyve (humaine), qui catalyse la phosphorylation du PtdIns $3 P$ en $\operatorname{PtdIns}(3,5) P_{2}$, se fixe au PtdIns3P via son domaine FYVE (Payrastre et al., 2001). Ainsi, un des rôles physiologiques du PtdIns3P est de servir de précurseur à la synthèse du $\operatorname{PtdIns}(3,5) P_{2}$.

Chez la levure $S$. cerevisiae, la délétion du gène VPS34 n'est pas létale mais elle entraîne une croissance très lente et une forte diminution de la résistance à de nombreux facteurs (température, $\mathrm{pH}$, éthanol, hygromycine $\mathrm{B}$, stress hyperosmotique, caféine ou rapamycine). La levure vps34 $\Delta$ présente également d'importants défauts de trafic membranaire qui se traduisent par une morphologie et un transport vacuolaire anormaux, l'excrétion de la carboxypeptidase Y (CPY) et un défaut d'autophagie (Kihara et al., 2001). Un des rôles essentiels de cette lipide kinase de levure est donc d'assurer l'intégrité du tri des protéines à destination de la vacuole en produisant du PtdIns3P, lequel permettra le recrutement de protéines effectrices du trafic (Henne et al., 2011).

\section{Le Ptdlns5P, un phosphoinositide méconnu}

\subsection{Synthèse du Ptdlns5P}

Le PtdIns5P est le PPIn mono-phosphate identifié le plus récemment (Rameh et al., 1997). Il est longtemps resté méconnu en raison de sa faible concentration basale dans les cellules de mammifères, mais également des difficultés liées aux techniques permettant de le séparer du PtdIns4P. En effet, en conditions basales dans les cellules de mammifères, le PtdIns5 $P$ représente moins de $10 \%$ des PPIn monophosphates (Payrastre et al., 2001).

Par ailleurs, aucune étude n'a mis en évidence la présence de PtdIns5P dans une souche sauvage de levure $S$. cerevisiae. En effet, Ymr1, l'unique représentante des myotubularines dans la levure, est dépourvue d'activité 3-phosphatase à l'encontre du PtdIns $(3,5) P_{2}$ (Taylor et al., 2000; Parrish et al., 2004).

Chez l'humain, le PtdIns5P est produit par la lipide kinase PIKfyve directement à partir du PtdIns (figure 1). In vivo, la surexpression de PIKfyve induit une augmentation de PtdIns5P, tandis que des souris hétérozygotes PIKfyve $+/$ null présentent des niveaux réduits de PtdIns5P en comparaison avec les souris témoins, sans pour autant que cela affecte la viabilité des souris mutantes (Ikonomov et al., 2011).

Du côté des lipides phosphatases, on retrouve les membres de la famille des myotubularines (MTM1, MTMR1-4,6,7) qui possèdent une activité 3 -phosphatase à l'encontre du $\operatorname{PtdIns}(3,5) P_{2}$, et génèrent ainsi du PtdIns5P (figure 1) (Laporte et al., 2003).

\subsection{Rôle physiologique du Ptdlns5P}

Le rôle du PtdIns5P dans les cellules de mammifères est encore relativement mal compris. Une fraction du PtdIns5 $P$ a été détectée au niveau du noyau et serait impliquée dans la réponse au stress, notamment en modulant l'activité du régulateur transcriptionnel 
ING2. ING2 est en outre la première protéine identifiée comme ayant un domaine de liaison spécifique au PtdIns5P, nommé PHD (Plant HomeoDomain finger), un domaine en doigt de zinc (Gozani et al., 2003).

\section{Le Ptdlns $(4,5) P_{2}$, un phosphoinositide impliqué dans l'endocytose}

\subsection{Synthèse du Ptdlns $(4,5) P_{2}$}

Chez la levure, le PtdIns $(4,5) P_{2}$ est présent en quantité équivalente au PtdIns4P, soit approximativement $30 \%$ des PPIn totaux et plus de $90 \%$ des différents PPIn bis-phosphorylés. Chez l'Homme, il représente environ $45 \%$ des PPIn totaux et plus de $90 \%$ des différents PPIn bis-phosphorylés. Le PtdIns $(4,5) P_{2}$ est donc un PPIn majoritaire (Di Paolo \& De Camilli, 2006).

Le PtdIns $(4,5) P_{2}$ est synthétisé par la PPIn 5-kinase Mss4 chez la levure (figure 1) et il est présent essentiellement à la membrane plasmique (figure 2) (Desrivières et al., 1998).

Chez l'Homme, plusieurs PPIn-kinases à l'origine du PtdIns $(4,5) P_{2}$ ont été identifiées : les PIP5K de type I $\alpha, \beta$ et $\gamma$ sont localisées à la membrane plasmique et convertissent le PtdIns $4 P$ en $\operatorname{PtdIns}(4,5) P_{2}$ (Ishihara et al., 1998); tandis que les PIP4K de type II sont situées au niveau de l'appareil de Golgi et convertissent le PtdIns5 $P$ en PtdIns $(4,5) P_{2}$ (Bunce et al., 2008; Clarke et al., 2008). Contrairement à la levure, les mammifères synthétisent également du PtdIns $(3,4,5) P_{3}$ qui peut servir de substrat à des PPIn 3 -phosphatases telles que PTEN (Phosphatase and TENsin homolog), TPIP $\alpha, \beta$ et $\gamma$ pour produire du $\operatorname{PtdIns}(4,5) P_{2}$ (figure 1) (Liu \& Bankaitis, 2010).

\subsection{Rôle physiologique du Ptdlns $(4,5) P_{2}$}

Chez $S$. cerevisiae et dans les cellules humaines, le $\operatorname{PtdIns}(4,5) P_{2}$ est majoritairement présent dans le feuillet cytoplasmique de la membrane plasmique (figure 2), où il agit comme un régulateur majeur du cytosquelette d'actine et de l'endocytose. Chez $S$. cerevisiae, Mss4 agit en combinaison avec la PtdIns 4-kinase Stt4 à la membrane plasmique pour générer le PtdIns $(4,5) P_{2}$ à partir de PtdIns. Ce PPIn est essentiel pour l'activation de la cascade de signalisation des MAP kinases médiée par Rho1/Pkc1. En effet, la localisation correcte de Rom2, la GEF (GTP Exchange Factor) de la GTPase Rho1, dépend directement de l'interaction entre son domaine $\mathrm{PH}$ et le $\operatorname{PtdIns}(4,5) P_{2}$ membranaire (Audhya \& Emr, 2002). L'organisation du cytosquelette d'actine dépend en grande partie de cette voie de signalisation (Desrivières et al., 1998). L'activité de Mss4 et la synthèse de $\operatorname{PtdIns(4,5)P_{2}}$ remplissent également une fonction essentielle dans l'endocytose qui dépend de l'actine chez la levure (Sun et al., 2007). En effet, les protéines effectrices de l'endocytose comme les domaines ENTH (Epsin $N$-Terminal Homology), ANTH (AP-180 N-Terminal Homology) ou PH (Pleckstrin Homology) possèdent un domaine de liaison au $\operatorname{PtdIns}(4,5) P_{2}$ (Itoh \& Takenawa, 2002; De Craene et al., 2012).

Chez l'Homme, le PtdIns $(4,5) P_{2}$ joue un rôle majeur dans la régulation de différentes voies de signalisation, notamment en raison des variations rapides auxquelles il peut être soumis par le jeu des kinases et phosphatases à PPIn (figure 1). À l'instar de celui de la levure, le PtdIns(4,5) $P_{2}$ remplit chez l'homme une fonction importante dans la régulation du cytosquelette d'actine. En effet, plusieurs études ont révélé des interactions entre le $\operatorname{PtdIns}(4,5) P_{2}$ et des protéines régulatrices de la polymérisation de l'actine. Dans les cellules de mammifères, le $\operatorname{PtdIns}(4,5) P_{2}$ est principalement requis au niveau de la membrane plasmique pour l'endocytose médiée par la clathrine (figure 2) (Di Paolo \& De Camilli, 2006). Il sert alors de signal de recrutement à de nombreuses protéines effectrices de l'étape d'internalisation de l'endocytose, via un domaine protéique (ENTH, ANTH, PH) liant spécifiquement le $\operatorname{PtdIns}(4,5) P_{2}$ (Itoh \& Takenawa, 2002; Di Paolo \& De Camilli, 2006; De Craene et al., 2012). Ainsi, le domaine ENTH de l'Epsine1 lie le PtdIns $(4,5) P_{2}$, et cette interaction induit un réarrangement structural de l'extrémité $\mathrm{N}$-terminale en une hélice- $\alpha$ qui va pénétrer dans la bicouche lipidique et générer la courbure membranaire nécessaire à la formation de la vésicule d'endocytose (Ford et al., 2001).

\section{Le Ptdlns $(3,5) P_{2}$, un phosphoinositide régulant le trafic endosome-lysosome}

\subsection{Synthèse du Ptdlns $(3,5) P_{2}$}

Le PtdIns(3,5) $P_{2}$ est également un PPIn peu abondant puisqu'il représente moins de $5 \%$ des PPIn totaux chez l'Homme et chez $S$. cerevisiae; il est enrichi au niveau des endosomes tardifs, du MVB et du lysosome/vacuole (figure 2) (Di Paolo \& De Camilli, 2006).

La levure $S$. cerevisiae synthétise séquentiellement le PtdIns $(3,5) P_{2}$, d'abord sous l'action de la PtdIns 3-kinase Vps34 qui génère le PtdIns3P, puis par phosphorylation du PtdIns3P en $\operatorname{PtdIns}(3,5) P_{2}$ par la PtdIns3P 5-kinase Fab1 (figure 1). Chez la levure, un stress hyperosmotique stimule très fortement la 


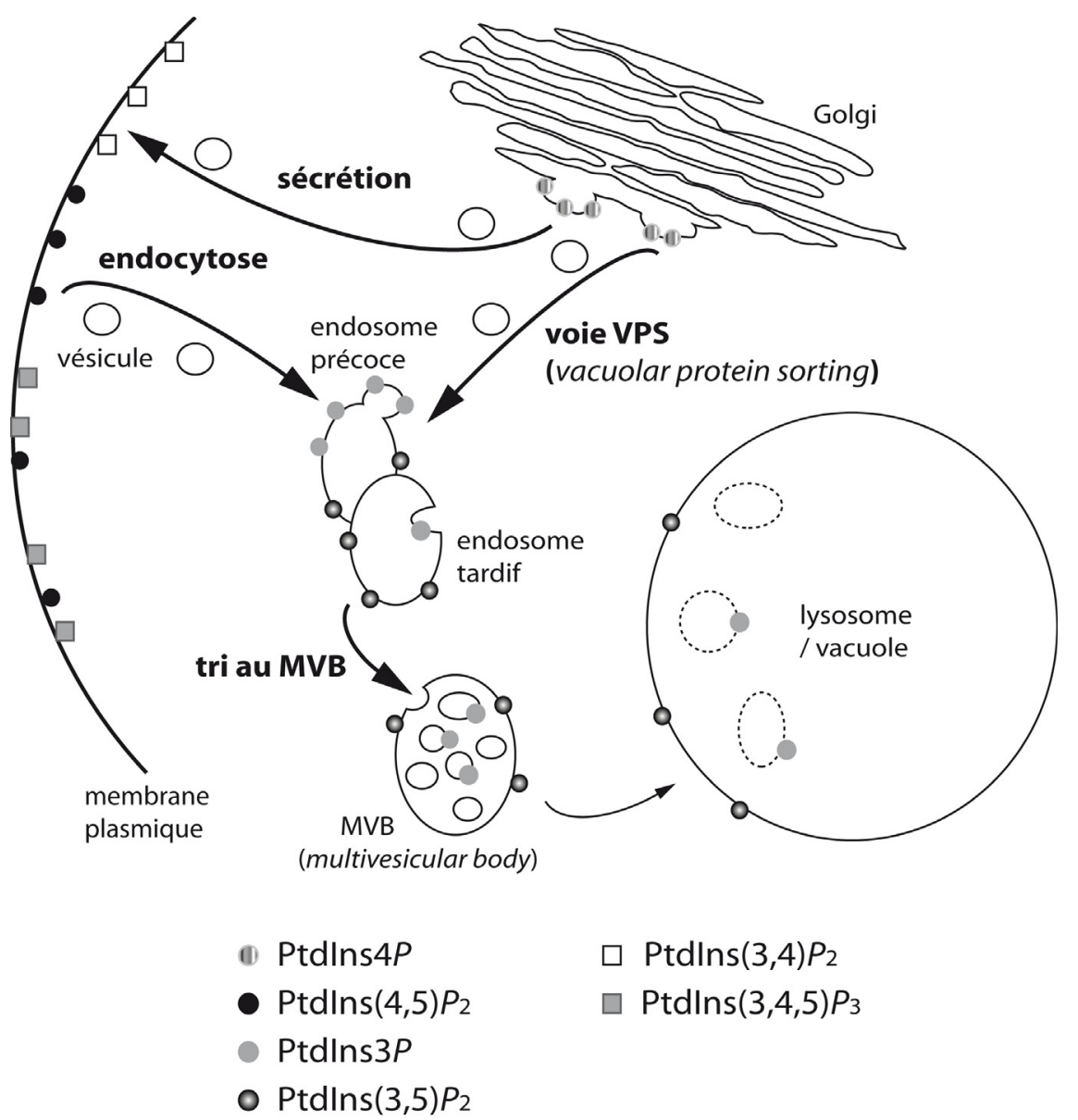

Fig. 2. Localisation intracellulaire des différents phosphoinositides et voies du trafic membranaire. Les différents phosphoinositides (PPIn) sont spécifiquement enrichis dans des membranes définies. Ils sont représentés par des symboles : en rond les PPIn impliqués dans le trafic intracellulaire avec les étapes qu'ils régulent; en carré les PPIn impliqués dans la signalisation cellulaire, ces deux types de PPIn sont absents chez la levure.

synthèse du PtdIns(3,5) $P_{2}$ qui voit son taux intracellulaire augmenter de 20 fois (Dove et al., 1997). La synthèse du PtdIns $(3,5) P_{2}$ est régulée par les protéines vacuolaires membranaires Vac7 et Vac14 (Bonangelino et al., 2002). En effet, dans des mutants affectés dans les gènes $F A B 1, V A C^{r} 7$ ou $V A C 14$, le $\operatorname{PtdIns}(3,5) P_{2}$ n'est pas détectable, et ce indépendamment des conditions osmotiques (Bonangelino et al., 2002). Si Vac7 et Vac14 sont indispensables à la synthèse de $\operatorname{PtdIns}(3,5) P_{2}$, elles n'interagissent pas puisque Vac7 est l'activateur majeur de Fab1 lors d'un choc hyperosmotique, alors que Vac14 agit dans le cadre d'un complexe avec la lipide phosphatase Fig4 pour réguler le renouvellement du $\operatorname{PtdIns}(3,5) P_{2}$ (Duex et al., $2006 \mathrm{~b})$. Ceci est très surprenant car la stimulation de la synthèse du $\operatorname{PtdIns}(3,5) P_{2}$ en réponse au stress osmotique est dépendante de réactions antagonistes de phosphorylation du $\operatorname{PtdIns}(3) P$ en $\operatorname{PtdIns}(3,5) P_{2}$ et de la déphosphorylation de celui-ci en PtdIns3P dans un cycle futile (Duex et al., 2006a). Il existe donc une interdépendance essentielle entre la lipide kinase et la phosphatase pour réguler le taux de PtdIns $(3,5) P_{2}$ mais également pour activer la production de $\operatorname{PtdIns}(3,5) P_{2}$ en réponse à un stimulus.

Chez l'Homme, la voie de synthèse du $\operatorname{PtdIns}(3,5) P_{2}$ est similaire à celle de la levure et c'est PIKfyve, l'unique homologue de Fab1, qui est à l'origine du $\operatorname{PtdIns}(3,5) P_{2}$ (figure 1) (Shisheva, 2008). A ce jour, aucune PPIn 4-phosphatase hydrolysant le PtdIns $(3,4,5) P_{3}$ n'a pu être mise en évidence. Par conséquent, il semblerait que l'unique voie de synthèse du PtdIns $(3,5) P_{2}$ passe par la phosphorylation du PtdIns3P (figure 1). De plus, la régulation de la synthèse du PtdIns $(3,5) P_{2}$ est conservée chez l'Homme, en effet PIKfyve interagit avec de multiples partenaires jouant un rôle dans l'homéostasie du PtdIns(3,5) $P_{2}$ (Shisheva, 2008). Par exemple, PIKfyve interagit avec son enzyme antagoniste Sac3/Fig4 
déphosphorylant spécifiquement le $\operatorname{PtdIns}(3,5) P_{2}$ en PtdIns3P. Cette interaction indirecte se fait par le biais de la protéine adaptatrice ArPIKfyve/Vac14 qui stabilise le complexe et stimule l'activité de PIKfyve (Ikonomov et al., 2010).

Une étude plus récente démontre clairement l'homologie dans la voie de synthèse du $\operatorname{PtdIns}(3,5) P_{2}$ entre la levure et les mammifères (Jin et al., 2008). En effet, que ce soit chez la levure ou la souris, la protéine Vac14 joue un rôle de plate-forme pour la régulation de la synthèse du PtdIns(3,5) $P_{2}$, en interagissant directement avec Fab1/PIKfyve, Fig4/Sac3 et Vac7 qui sont les effecteurs de cette voie de synthèse. Ainsi Vac7, Fab1 et Fig4 pourront entrer en contact par l'intermédiaire de leur liaison à différentes régions de Vac14 (Jin et al., 2008).

\subsection{Rôle physiologique du Ptdlns(3,5) $P_{2}$}

Différentes études ont montré le rôle du $\operatorname{PtdIns}(3,5) P_{2}$ dans la régulation du trafic entre les endosomes et la vacuole (l'équivalent du lysosome chez la levure) (figure 2).

Chez $S$. cerevisiae, les cellules fab1 $\Delta$ présentent un défaut de croissance à $23{ }^{\circ} \mathrm{C}$, ne sont pas viables à $37^{\circ} \mathrm{C}$ et montrent un défaut d'acidification de la vacuole. Elles montrent aussi d'importants défauts d'homéostasie vacuolaire avec un compartiment vacuolaire anormalement élargi, occupant jusqu'à $80 \%$ du volume de la cellule. Cet encombrement lié à la taille de la vacuole peut également causer une distribution incorrecte des chromosomes lors de la division cellulaire (Yamamoto et al., 1995). La présence de Vac7 et de Vac14, qui régulent la synthèse du $\operatorname{PtdIns}(3,5) P_{2}$ par Fab1, est requise pour permettre le maintien de la morphologie vacuolaire ainsi que pour la transmission correcte de la vacuole de la cellule mère à la cellule fille (Bonangelino et al., 2002).

Le $\operatorname{PtdIns}(3,5) P_{2}$ joue un rôle essentiel dans le tri des protéines membranaires au niveau des endosomes tardifs/MVB (Odorizzi et al., 1998). Les protéines membranaires à destination de la vacuole sont marquées par de l'ubiquitine au niveau des endosomes. Ces cargos ubiquitinés sont reconnus successivement par les complexes ESCRT-0 à -2 pour leur internalisation dans les vésicules internes de l'endosome afin de former le MVB, dont la fusion avec la vacuole aboutit au largage des vésicules dans la lumière de la vacuole (Gruenberg \& Stenmark, 2004). Au niveau des endosomes, les epsines de levure Ent3 et Ent5 interagissent avec le $\operatorname{PtdIns}(3,5) P_{2}$ grâce à leur domaine ENTH, et elles sont indispensables pour le tri des cargos au MVB (Friant et al., 2003; Eugster et al., 2004). La protéine liant le plus spécifiquement et avec le plus d'affinité le $\operatorname{PtdIns}(3,5) P_{2}$ est à l'heure actuelle la protéine de levure Svp1/Atg18, qui joue un rôle dans l'autophagie chez la levure. De manière intéressante, Svp1/Atg18 régule aussi l'activité de Fab1, en se liant également à la protéine régulatrice Vac7 (elle-même recrutée par la protéine plate-forme Vac14). Ainsi, Svp1/Atg18 agirait comme un senseur du taux de $\operatorname{PtdIns}(3,5) P_{2}$ en permettant la régulation de sa synthèse via un rétrocontrôle impliquant Vac7 et Vac14 (Efe et al., 2007).

Chez la souris, le mutant PIKfyve $\mathrm{KO}^{\mathrm{KO}}$ meurt très tôt au cours du développement embryonnaire (Ikonomov et al., 2011), ce qui témoigne de l'importance fondamentale de ce lipide membranaire dans les processus cellulaires. Fab1 et PIKfyve possèdent toutes les deux un domaine FYVE capable de lier le PtdIns3P. Cette interaction avec le PtdIns3P va entraîner leur recrutement aux endosomes, où le PtdIns3P est enrichi, et permettre la synthèse du $\operatorname{PtdIns}(3,5) P_{2}$ (figure 2) (Sbrissa et al., 2002). PIKfyve a été décrite comme ayant un rôle dans divers processus tels que le tri endosomal des protéines, l'homéostasie vacuolaire/lysosomale ou la régulation de voies de signalisation (Payrastre et al., 2001).

\section{Le Ptdlns $(3,4) P_{2}$, un second messager lipidique}

\subsection{Synthèse du Ptdlns $(3,4) P_{2}$}

Chez la levure $S$. cerevisiae, aucune étude n'a pu mettre en évidence la présence du PtdIns $(3,4) P_{2}$. Chez l'Homme, le PtdIns(3,4) $P_{2}$ est essentiellement localisé à la membrane plasmique (figure 2) et compte pour moins de $10 \%$ des PPIn totaux en condition basale. Cependant, ces niveaux peuvent transitoirement augmenter en réponse à une stimulation par des facteurs de croissance ou des cytokines (Payrastre et al., 2001). Le PtdIns(3,4) $P_{2}$ est obtenu majoritairement par la phosphorylation du PtdIns4P en $\operatorname{PtdIns}(3,4) P_{2}$ par les PI3K II (figure 1). La déphosphorylation du $\operatorname{PtdIns}(3,4,5) P_{3}$ en $\operatorname{PtdIns}(3,4) P_{2}$ peut être réalisée par les PPIn 5-phosphatases SHIP1/INPP5D, SHIP2/INPPL1, OCRL1, INPP5B ainsi que par les Synaptojanines 1 et 2 (figure 1) (Liu \& Bankaitis, 2010).

\subsection{Rôle physiologique du Ptdlns $(3,4) P_{2}$}

Plusieurs études suggèrent que le $\operatorname{PtdIns}(3,4) P_{2}$ fonctionne comme un second messager. En effet, il est par exemple capable de recruter les protéine-kinases Akt et PDK1 via leurs domaines PH. Le lien entre le $\operatorname{PtdIns}(3,4) P_{2}$ et la voie de signalisation PI3K/Akt suggère que ce PPIn pourrait être impliqué dans un grand nombre de processus biologiques comme le contrôle du cycle cellulaire, la survie, l'angiogenèse, la 
prolifération ou le métabolisme du glucose. L'équilibre entre le $\operatorname{PtdIns}(3,4) P_{2}$ et le $\operatorname{PtdIns}(3,4,5) P_{3}$ par le jeu des lipides kinases et phosphatases (figure 1) pourrait donc jouer un rôle essentiel dans la régulation des voies de signalisation en aval de Akt (Hers et al., 2011). Malgré les nombreuses études sur ce PPIn, sa fonction à la membrane plasmique est peu documentée, en effet la plupart des études se focalisent sur le $\operatorname{PtdIns}(3,4,5) P_{3}$.

Parmi les différents domaines protéiques, seuls les domaines PH de la protéine TAPP1 (tandem $P H$ domain containing protein 1) interagissent spécifiquement avec le $\operatorname{PtdIns}(3,4) P_{2}$. La protéine TAPP1 régule l'organisation du cytosquelette d'actine. Une étude suggère que la liaison de TAPP1 au $\operatorname{PtdIns}(3,4) P_{2}$ favoriserait le recrutement à la membrane plasmique de PTPL1 (Protein Tyrosine Phosphatase Like protein 1, ou FAP-1), une tyrosine phosphatase ubiquitaire impliquée dans la survie cellulaire (Kimber et al., 2003).

\section{Le Ptdlns $(3,4,5) P_{3}$, un effecteur clé de la voie de signalisation $\mathrm{PI} / \mathrm{K} / \mathrm{Akt}$}

\subsection{Synthèse du Ptdlns $(3,4,5) P_{3}$}

La levure $S$. cerevisiae ne présente pas de taux détectable de $\operatorname{PtdIns}(3,4,5) P_{3}$ et celui-ci est donc considéré comme absent de cet organisme. Chez l'Homme, le PtdIns $(3,4,5) P_{3}$ représente moins de $5 \%$ des PPIn totaux et il est quasiment indétectable dans des cellules quiescentes. Ses niveaux intracellulaires peuvent cependant augmenter de façon rapide et transitoire, jusqu'à 100 fois en réponse à un agoniste (Milne et al., 2005). Le $\operatorname{PtdIns}(3,4,5) P_{3}$ est essentiellement synthétisé à la membrane plasmique (figure 2) par les PPIn 3-kinases de classe I à partir du PtdIns $(4,5) P_{2}$ (figure 1), mais il n'est pas exclu que des pools minoritaires de $\operatorname{PtdIns}(3,4,5) P_{3}$ puissent exister à la membrane de compartiments intracellulaires en réponse à un agoniste (Payrastre et al., 2001).

La synthèse du $\operatorname{PtdIns}(3,4,5) P_{3}$ est finement régulée, étant donné que cette molécule signal est au centre de nombreuses voies de signalisation. Parmi les régulateurs du taux intracellulaire de $\operatorname{PtdIns}(3,4,5) P_{3}$, on retrouve la phosphatase PTEN qui catalyse la déphosphorylation en position 3 du $\operatorname{PtdIns}(3,4,5) P_{3}$ pour produire du $\operatorname{PtdIns}(4,5) P_{2}$. PTEN a été caractérisée comme un suppresseur de tumeur et des mutations dans PTEN sont impliquées dans de nombreux cancers (Liu \& Bankaitis, 2010). Le rôle principal de PTEN est de réguler le cycle cellulaire et l'apoptose via son activité phosphatase requise pour la régulation de la voie de signalisation dépendant de la kinase Akt.

\subsection{Rôle physiologique du Ptdlns $(3,4,5) P_{3}$}

Bien qu'il soit présent à de très faibles taux, le PtdIns $(3,4,5) P_{3}$ est sans doute le PPIn dont le rôle est le mieux caractérisé. En effet, ses effecteurs impliqués dans différentes voies de signalisation le lient via leurs domaines $\mathrm{PH}$, et sa dérégulation entraîne le développement de nombreux cancers. Parmi les effecteurs, il y a des facteurs d'échange de petites protéines $\mathrm{G}$ de la famille Arf (ADP-ribosylation factors). D'autres effecteurs sont les sérine-thréonine kinases PDK1 (Phosphoinositide Dependent Kinase 1) et Akt ainsi que la phospholipase $\mathrm{C} \gamma(\mathrm{PLC} \gamma)$ qui font ainsi le lien entre ce PPIn et le contrôle de fonctions cellulaires telles que la prolifération et la survie cellulaire, la dynamique du cytosquelette, la mobilité, le trafic membranaire et l'apoptose (Lemmon, 2003).

Un des rôles les plus étudiés du PtdIns $(3,4,5) P_{3}$ est celui de régulateur de la kinase Akt qui lie ce PPIn via son domaine $\mathrm{PH}$. Akt joue un rôle très important car d'une part elle va permettre l'activation de la PI3K I et la synthèse du $\operatorname{PtdIns}(3,4,5) P_{3}$, et d'autre part sa liaison au PtdIns $(3,4,5) P_{3}$ via son domaine $\mathrm{PH}$ va lui permettre d'être ancrée à la membrane plasmique, où elle sera phosphorylée et activée par la protéine kinase PDK1 (Hers et al., 2011). Chez les mammifères, il existe trois isoformes d'Akt nommées Akt1, Akt2 et Akt3 qui sont activées par des facteurs de croissance ou d'autres stimuli extracellulaires, ainsi que par des mutations oncogéniques dans différents régulateurs d'Akt (Ras, les sous-unités p110 et p85 de la PI3K I et PTEN). En effet, le domaine PH d'Akt lie aussi le $\operatorname{PtdIns}(3,4) P_{2}$ qui est produit par PTEN à partir du PtdIns $(3,4,5) P_{3}$ (figure 1) (Hers et al., 2011). La voie de signalisation $\operatorname{PtdIns}(3,4,5) P_{3} / \operatorname{PtdIns}(3,4) P_{2} /$ Akt est impliquée dans la régulation de nombreuses fonctions biologiques dont la prolifération cellulaire, la survie et le métabolisme. La dérégulation d'Akt aboutit à des cancers, des diabètes et des maladies cardiovasculaires et neurologiques. Il est donc très important de trouver des inhibiteurs agissant sur Akt pour traiter ces pathologies. Ainsi, parmi les inhibiteurs couramment utilisés en thérapie, beaucoup agissent sur la liaison entre les PPIn et le domaine PH d'Akt (Hers et al., 2011).

\section{Conclusion}

Les phosphoinositides sont des molécules lipidiques qui coordonnent le trafic intracellulaire. Les différentes formes phosphorylées des PPIn sont contrôlées par un jeu de kinases et de phosphatases en réponse à des stimuli. Les sept PPIn sont présents en quantité variable dans les cellules et certaines espèces sont enrichies dans des compartiments intracellulaires bien précis. Les PPIn sont tous virtuellement inter-convertibles 
sous réserve que l'organisme possède le jeu d'enzymes appropriées. Ainsi, le PtdIns5 $P$, le $\operatorname{PtdIns}(3,4) P_{2}$ et le $\operatorname{PtdIns}(3,4,5) P_{3}$, présents chez les mammifères, n'ont pu être mis en évidence dans la levure $S$. cerevisiae. Les PPIn jouent un rôle essentiel dans l'identité des membranes intracellulaires. De plus, des changements locaux dans les niveaux de PPIn permettent une régulation spatiotemporelle de divers événements tels que le bourgeonnement vésiculaire, la fusion membranaire ou la dynamique du trafic intracellulaire. $\mathrm{Du}$ fait de leur faible abondance, moins de $10 \%$ des phospholipides cellulaires, les PPIn peuvent être soumis localement à des variations très fortes dans leur concentration; c'est plus particulièrement le cas pour le $\operatorname{PtdIns}(3,4,5) P_{3}$ en réponse à une stimulation cellulaire chez les mammifères et le $\operatorname{PtdIns}(3,5) P_{2}$ en réponse à un choc osmotique chez la levure. Ainsi de nombreuses études se sont penchées sur le métabolisme des PPIn, leurs localisations et leurs rôles intracellulaires. En effet, malgré leur faible proportion, ils jouent un rôle essentiel dans le recrutement et/ou l'activation de protéines effectrices et interviennent dans la régulation de différentes fonctions cellulaires.

Remerciements. Ce travail a bénéficié du soutien du CNRS (ATIP-CNRS 05-00932 et ATIP-Plus 2008-3098 à S.F.), de la Fondation pour la Recherche Médicale (FRM INE20051105238 et FRM-Comité Alsace 2006CX67-1 à S.F. et bourse post-doctorale FRM à J-O.D.C.), de l'Agence Nationale de la Recherche (ANR-07-BLAN-0065 et ANR-13-BSV2-0004 à S.F.), de l'Association Française contre les Myopathies (AFM-SB/CP/2013-0133/16551 à S.F. et financement de thèse à D.L.B et M.R.) et du programme IDEX de l'Université de Strasbourg (financement de thèse à M.S-V.). Nous remercions aussi nos collaborateurs Bernard Payrastre (I2MC, Toulouse), Jocelyn Laporte et Jean-Louis Mandel (IGBMC, Illkirch) pour leur aide précieuse, et Bruno Goud (Institut Curie, Paris) pour son soutien sans faille.

\section{Références}

Antonsson, B.E. (1994). Purification and characterization of phosphatidylinositol synthase from human placenta. Biochem J, 297, 517-522.

Audhya, A., and Emr, S.D. (2002). Stt4 PI 4-kinase localizes to the plasma membrane and functions in the Pkc1-mediated MAP kinase cascade. Dev Cell, 2, 593605.

Audhya, A., Foti, M., and Emr, S.D. (2000). Distinct roles for the yeast phosphatidylinositol 4-kinases, Stt4p and Pik1p, in secretion, cell growth, and organelle membrane dynamics. Mol Biol Cell, 11, 2673-2689.

Bonangelino, C.J., Nau, J.J., Duex, J.E., Brinkman, M., Wurmser, A.E., Gary, J.D., Emr, S.D., and Weisman, L.S. (2002). Osmotic stress-induced increase of phosphatidylinositol 3,5-bisphosphate requires Vac14p, an activator of the lipid kinase Fab1p. J Cell Biol, 156, 1015-1028.

Bunce, M.W., Boronenkov, I.V., and Anderson, R.A. (2008). Coordinated activation of the nuclear ubiquitin ligase Cul3-SPOP by the generation of phosphatidylinositol 5-phosphate. J Biol Chem, 283, 8678-8686.

Clarke, J.H., Emson, P.C., and Irvine, R.F. (2008). Localization of phosphatidylinositol phosphate kinase IIgamma in kidney to a membrane trafficking compartment within specialized cells of the nephron. Am J Physiol Renal Physiol, 295, F1422-1430.

De Craene, J.O., Ripp, R., Lecompte, O., Thompson, J.D., Poch, O., and Friant, S. (2012). Evolutionary analysis of the ENTH/ANTH/VHS protein superfamily reveals a coevolution between membrane trafficking and metabolism. BMC Genomics, 13, 297.

De Matteis, M., Godi, A., and Corda, D. (2002). Phosphoinositides and the Golgi complex. Curr Opin Cell Biol, 14, 434-447.

Desrivières, S., Cooke, F.T., Parker, P.J., and Hall, M.N. (1998). MSS4, a phosphatidylinositol-4-phosphate 5kinase required for organization of the actin cytoskeleton in Saccharomyces cerevisiae. J Biol Chem, 273, 15787-15793.

Di Paolo, G., and De Camilli, P. (2006). Phosphoinositides in cell regulation and membrane dynamics. Nature, 443, 651-657.

Dove, S.K., Cooke, F.T., Douglas, M.R., Sayers, L.G., Parker, P.J., and Michell, R.H. (1997). Osmotic stress activates phosphatidylinositol-3,5-bisphosphate synthesis. Nature, 390, 187-192.

Duex, J.E., Nau, J.J., Kauffman, E.J., and Weisman, L.S. (2006a). Phosphoinositide 5-phosphatase Fig 4p is required for both acute rise and subsequent fall in stressinduced phosphatidylinositol 3,5-bisphosphate levels. Eukaryot Cell, 5, 723-731.

Duex, J.E., Tang, F., and Weisman, L.S. (2006b). The Vac14p-Fig4p complex acts independently of Vac7p and couples PI3,5P2 synthesis and turnover. J Cell Biol, 172, 693-704.

Efe, J.A., Botelho, R.J., and Emr, S.D. (2007). Atg18 regulates organelle morphology and Fab1 kinase activity independent of its membrane recruitment by phosphatidylinositol 3,5-bisphosphate. Mol Biol Cell, 18, 4232-4244.

Eugster, A., Pécheur, E.I., Michel, F., Winsor, B., Letourneur, F., and Friant, S. (2004). Ent5p is required with Ent3p and Vps27p for ubiquitin-dependent protein sorting into the multivesicular body. Mol Biol Cell, 15, 3031-3041.

Ford, M.G., Pearse, B.M., Higgins, M.K., Vallis, Y., Owen, D.J., Gibson, A., Hopkins, C.R., Evans, P.R., and McMahon, H.T. (2001). Simultaneous binding of PtdIns(4,5)P2 and clathrin by AP180 in the nucleation of clathrin lattices on membranes. Science, 291, 10511055 .

Friant, S., Pécheur, E.I., Eugster, A., Michel, F., Lefkir, Y., Nourrisson, D., and Letourneur, F. (2003). Ent3p 
Is a PtdIns(3,5)P2 effector required for protein sorting to the multivesicular body. Dev Cell, 5, 499-511.

Gary, J.D., Sato, T.K., Stefan, C.J., Bonangelino, C.J., Weisman, L.S., and Emr, S.D. (2002). Regulation of Fab1 phosphatidylinositol 3-phosphate 5-kinase pathway by Vac7 protein and Fig4, a polyphosphoinositide phosphatase family member. Mol Biol Cell, 13, 1238-1251.

Gillooly, D.J., Morrow, I.C., Lindsay, M., Gould, R., Bryant, N.J., Gaullier, J.M., Parton, R.G., and Stenmark, H. (2000). Localization of phosphatidylinositol 3-phosphate in yeast and mammalian cells. EMBO $J, 19,4577-4588$.

Godi, A., Di Campli, A., Konstantakopoulos, A., Di Tullio, G., Alessi, D.R., Kular, G.S., Daniele, T., Marra, P., Lucocq, J.M., and De Matteis, M.A. (2004). FAPPs control Golgi-to-cell-surface membrane traffic by binding to ARF and PtdIns(4)P. Nat Cell Biol, 6, 393-404.

Gozani, O., Karuman, P., Jones, D.R., Ivanov, D., Cha, J., Lugovskoy, A.A., Baird, C.L., Zhu, H., Field, S.J., Lessnick, S.L., Villasenor J., Mehrotra B., Chen J., Rao V.R., Brugge J.S., Ferguson C.G., Payrastre B., Myszka D.G., Cantley L.C., Wagner G., Divecha N., Prestwich GD., and Yuan J. (2003). The PHD finger of the chromatin-associated protein ING2 functions as a nuclear phosphoinositide receptor. Cell , 114, 99-111.

Gruenberg, J., and Stenmark, H. (2004). The biogenesis of multivesicular endosomes. Nat Rev Mol Cell Biol, 5, 317-323.

Henne, W.M., Buchkovich, N.J., and Emr, S.D. (2011). The ESCRT pathway. Dev Cell, 21, 77-91.

Herman, P.K., and Emr, S.D. (1990). Characterization of VPS34, a gene required for vacuolar protein sorting and vacuole segregation in Saccharomyces cerevisiae. Mol Cell Biol, 10, 6742-6754.

Hers, I., Vincent, E.E., and Tavare, J.M. (2011). Akt signalling in health and disease. Cell Signal, 23, 1515-1527.

Ikonomov, O.C., Sbrissa, D., Delvecchio, K., Xie, Y., Jin, J.P., Rappolee, D., and Shisheva, A. (2011). The phosphoinositide kinase PIKfyve is vital in early embryonic development: preimplantation lethality of PIKfyve-/embryos but normality of PIKfyve+/- mice. J Biol Chem, 286, 13404-13413.

Ikonomov, O.C., Sbrissa, D., Fligger, J., Delvecchio, K., and Shisheva, A. (2010). ArPIKfyve regulates Sac3 protein abundance and turnover: disruption of the mechanism by Sac3I41T mutation causing Charcot-Marie-Tooth 4J disorder. J Biol Chem, 285, 26760-26764.

Ishihara, H., Shibasaki, Y., Kizuki, N., Wada, T., Yazaki, Y., Asano, T., and Oka, Y. (1998). Type I phosphatidylinositol-4-phosphate 5-kinases. Cloning of the third isoform and deletion/substitution analysis of members of this novel lipid kinase family. J Biol Chem, 273, 8741-8748.

Itoh, T., and Takenawa, T. (2002). Phosphoinositidebinding domains: Functional units for temporal and spatial regulation of intracellular signalling. Cell Signal, 14, 733-743.

Jin, N., Chow, C.Y., Liu, L., Zolov, S.N., Bronson, R., Davisson, M., Petersen, J.L., Zhang, Y., Park, S., Duex, J.E., Goldowitz D., Meisler M.H., and Weisman L.S. (2008). VAC14 nucleates a protein complex essential for the acute interconversion of PI3P and $\mathrm{PI}(3,5) \mathrm{P}(2)$ in yeast and mouse. EMBO J, 27, 3221-3234.

Kihara, A., Noda, T., Ishihara, N., and Ohsumi, Y. (2001). Two distinct Vps34 phosphatidylinositol 3-kinase complexes function in autophagy and carboxypeptidase Y sorting in Saccharomyces cerevisiae. J Cell Biol, 152, 519-530.

Kimber, W.A., Deak, M., Prescott, A.R., and Alessi, D.R. (2003). Interaction of the protein tyrosine phosphatase PTPL1 with the PtdIns(3,4)P2-binding adaptor protein TAPP1. Biochem J, 376, 525-535.

Laporte, J., Bedez, F., Bolino, A., and Mandel, J.L. (2003). Myotubularins, a large disease-associated family of cooperating catalytically active and inactive phosphoinositides phosphatases. Hum Mol Genet, 12 Spec No 2, R285-292.

Lecompte, O., Poch, O., and Laporte, J. (2008). PtdIns5P regulation through evolution: roles in membrane trafficking? Trends Biochem Sci, 33, 453-460.

Lemmon, M.A. (2003). Phosphoinositide recognition domains. Traffic, 4, 201-213.

Levine, T.P., and Munro, S. (2002). Targeting of Golgispecific pleckstrin homology domains involves both PtdIns 4-kinase-dependent and -independent components. Curr Biol, 12, 695-704.

Liu, Y., and Bankaitis, V.A. (2010). Phosphoinositide phosphatases in cell biology and disease. Prog Lipid Res, 49, 201-217.

Marcus, A.J., Ullman, H.L., and Safier, L.B. (1969). Lipid composition of subcellular particles of human blood platelets. J Lipid Res, 10, 108-114.

Milne, S.B., Ivanova, P.T., DeCamp, D., Hsueh, R.C., and Brown, H.A. (2005). A targeted mass spectrometric analysis of phosphatidylinositol phosphate species. $J$ Lipid Res, 46, 1796-1802.

Mizuno-Yamasaki, E., Medkova, M., Coleman, J., and Novick, P. (2010). Phosphatidylinositol 4-phosphate controls both membrane recruitment and a regulatory switch of the Rab GEF Sec2p. Dev Cell, 18, 828-840.

Nikawa, J., and Yamashita, S. (1984). Molecular cloning of the gene encoding CDPdiacylglycerol-inositol 3phosphatidyl transferase in Saccharomyces cerevisiae. Eur J Biochem, 143, 251-256.

Odorizzi, G., Babst, M., and Emr, S.D. (1998). Fab1p PtdIns(3)P 5-kinase function essential for protein sorting in the multivesicular body. Cell, 95, 847-858.

Panaretou, C., Domin, J., Cockcroft, S., and Waterfield, M.D. (1997). Characterization of p150, an adaptor protein for the human phosphatidylinositol (PtdIns) 3kinase. Substrate presentation by phosphatidylinositol transfer protein to the p150.Ptdins 3-kinase complex. J Biol Chem, 272, 2477-2485. 
Parrish, W.R., Stefan, C.J., and Emr, S.D. (2004). Essential role for the myotubularin-related phosphatase Ymr1p and the synaptojanin-like phosphatases Sj12p and Sj13p in regulation of phosphatidylinositol 3-phosphate in yeast. Mol Biol Cell, 15, 3567-3579.

Payrastre, B., Missy, K., Giuriato, S., Bodin, S., Plantavid, M., and Gratacap, M. (2001). Phosphoinositides: key players in cell signalling, in time and space. Cell Signal, $13,377-387$.

Rameh, L.E., Tolias, K.F., Duckworth, B.C., and Cantley, L.C. (1997). A new pathway for synthesis of phosphatidylinositol-4,5-bisphosphate. Nature, 390, 192-196.

Sbrissa, D., Ikonomov, O.C., Deeb, R., and Shisheva, A. (2002). Phosphatidylinositol 5-phosphate biosynthesis is linked to PIKfyve and is involved in osmotic response pathway in mammalian cells. J Biol Chem, 277, 47276-47284.

Schu, P.V., Takegawa, K., Fry, M.J., Stack, J.H., Waterfield, M.D., and Emr, S.D. (1993). Phosphatidylinositol 3-kinase encoded by yeast VPS34 gene essential for protein sorting. Science, 260, 88-91.

Shisheva, A. (2008). PIKfyve: Partners, significance, debates and paradoxes. Cell Biol Int, 32, 591-604.

Slessareva, J.E., Routt, S.M., Temple, B., Bankaitis, V.A., and Dohlman, H.G. (2006). Activation of the phosphatidylinositol 3-kinase Vps34 by a G protein alpha subunit at the endosome. Cell, 126, 191-203.
Spector, A.A., and Yorek, M.A. (1985). Membrane lipid composition and cellular function. J Lipid Res, 26, 1015-1035.

Sun, Y., Carroll, S., Kaksonen, M., Toshima, J.Y., and Drubin, D.G. (2007). PtdIns(4,5)P2 turnover is required for multiple stages during clathrin- and actindependent endocytic internalization. J Cell Biol, 177, 355-367.

Taylor, G.S., Maehama, T., and Dixon, J.E. (2000). Inaugural article: myotubularin, a protein tyrosine phosphatase mutated in myotubular myopathy, dephosphorylates the lipid second messenger, phosphatidylinositol 3-phosphate. Proc Natl Acad Sci USA, 97, 8910-8915.

Trevelyan, W.E. (1966). Preparation of phosphatidyl inositol from baker's yeast. J Lipid Res, 7, 445-447.

Vanhaesebroeck, B., Leevers, S.J., Ahmadi, K., Timms, J., Katso, R., Driscoll, P.C., Woscholski, R., Parker, P.J., and Waterfield, M.D. (2001). Synthesis and function of 3-phosphorylated inositol lipids. Annu Rev Biochem, 70, 535-602.

Yamamoto, A., DeWald, D.B., Boronenkov, I.V., Anderson, R.A., Emr, S.D., and Koshland, D. (1995). Novel PI(4)P 5-kinase homologue, Fab1p, essential for normal vacuole function and morphology in yeast. Mol Biol Cell, 6, 525-539.

Zinser, E., Sperka-Gottlieb, C.D., Fasch, E.V., Kohlwein, S.D., Paltauf, F., and Daum, G. (1991). Phospholipid synthesis and lipid composition of subcellular membranes in the unicellular eukaryote Saccharomyces cerevisiae. J Bacteriol, 173, 2026-2034. 